\title{
AN AUTOMATED CALCULATION OF PLANT ROOT DISTRIBUTION PARAMETERS BASED ON ROOT LENGTH DENSITY DATA
}

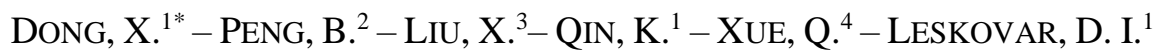 \\ ${ }^{I}$ Texas A\&M AgriLife Research and Extension Center at Uvalde, Uvalde TX 78801, USA \\ ${ }^{2}$ Yancheng Institute of Technology, Yancheng City, 224051 Jiangsu Province, China \\ ${ }^{3}$ Noble Research Institute, Ardmore OK 73401, USA \\ ${ }^{4}$ Texas A\&M AgriLife Research and Extension Center at Amarillo, Amarillo TX 79106, USA \\ *Corresponding author \\ e-mail: xuejun.dong@ag.tamu.edu \\ (Received $11^{\text {th }}$ Oct 2018; accepted $22^{\text {nd }}$ Nov 2018)
}

\begin{abstract}
Pattern of vertical distribution of plant roots determines plant water and nutrient uptake and influences various soil processes under field conditions. However, information of root distribution in the whole rooting profile is rarely available in practice. This paper documents a method to quickly quantify root vertical distribution pattern using limited root length density data obtained from soil cores under field conditions. It was implemented through the development of a Minitab macro and an R script, with sample calculations conducted on field collected root length density data from the winter wheat crop in Texas, USA. The method is potentially useful for quantifying plant root activities in natural soils where deep roots are difficult to access by physical means and data of root length density distribution are only available in the upper root zone.
\end{abstract}

Keywords: computer code, data processing, field measurement, rooting depth, soil core

\section{Introduction}

Vertical distribution of roots of land plants generally follows an exponential decay pattern (Weaver, 1926) and this has been frequently recognized in models describing plant water uptake from the soil profile (Ojha and Rai, 1996; Wu et al., 1999; Yadav et al., 2009; Dong et al., 2010; Dong, 2016). This fashion of root distribution is also an important trait dictating plant resource acquisition from the soil environment (Xue et al., 2003; Acuña and Wade, 2013; Sharma et al., 2014; Wasson et al., 2014; Hodgkinson et al., 2017). For example, the depth at which the densest roots locate in the soil profile influences plants' capacity to tolerate drought stress (Wan et al., 2000; Yu et al., 2007). The roots of numerous plants can easily reach 2 meters deep in the soil (Coupland and Johnson, 1965), but it is difficult to measure the roots from deeper soil profile. As a result, field studies usually only report root data collected from upper soil layers (Xue et al., 2003), and a major challenge for researchers is to extract the most information from the limited survey data of plant roots. The need for obtaining simple indices to summarize root distribution becomes more acute for large field experiment involving a complex design structure. The pattern of vertical distribution of plant roots in the soil profile is described elsewhere (Wu et al., 1999; Zuo et al., 2004; Yadav et al., 2009; Zuo et al., 2013). Despite complexities due to variations in plant growth and soil microenvironment, a salient feature pervading the literature is the exponential decay of the roots with the increase of soil depth. This fashion of root distribution allows calculation 
of the normalized root length density (Wu et al., 1999) that has important applications in describing water uptake by plant roots. The main purpose of this paper is to describe a simple procedure for estimating root distribution pattern using limited field data. This will include an automated calculation to extract root information from large survey data sets, so that variables such as normalized root length density can easily be obtained based on field survey data of root length density distribution.

\section{Method}

The relative importance of roots located in a particular soil layer to plant water uptake is conveniently described by the normalized root length density $L_{n r d}$ (dimensionless), which can be calculated according to Wu et al. (1999):

$$
L_{\text {nrd }}=\frac{L_{d}\left(z_{r}\right)}{\int_{0}^{1} L_{d}\left(z_{r}\right) d z_{r}}
$$

where $L_{d}\left(z_{r}\right)$ is root length density $\left(\mathrm{cm} \mathrm{cm}^{-3}\right), z_{r}=z_{/} / L_{m}$ is the relative soil depth containing plant roots and $z(\mathrm{~cm})$ and $L_{m}$ are soil depth and the maximum rooting depth $(\mathrm{cm})$, respectively. It has been shown that Equation 1 can be used as a weighting factor to partition potential transpiration into components of root water uptake occurring from different soil layers (Wu et al., 1999; Zuo et al., 2013).

Under field conditions, values of $L_{m}$ at different growth stages of a crop may be estimated using a sigmoidal growth curve (Wu et al., 1999; Dong et al., 2010), or based on the ratio of potential transpiration and measured maximum rooting depth at the peak growth stage (Ojha and Rai, 1996). While $L_{d}\left(z_{r}\right)$ can easily be measured for a particular soil layer by destructive sampling, the denominator of Equation 1 normally needs to be estimated based on measured root length density data, as well as a model describing root length density as a function of soil depth. Assuming that root length density $L_{d}(z)$ is measured for the first $L^{\prime} \mathrm{cm}$ of soil for $n$ intervals, each of $L^{\prime} / n \mathrm{~cm}$ in depth, root length density as a function of soil depth may be described using an exponential decay function

$$
L_{d}(z)=K e^{-\alpha z}
$$

in which $K$ and $\alpha$ are parameters that can be estimated using a nonlinear least-squares regression procedure. Then, with the estimated values of $K$ and $\alpha$, the total lengths of roots (per unit of soil surface area) contained in the soil depth from $L^{\prime}$ to $L_{m}$ can be estimated by integrating Equation 2:

$$
L_{\text {bottom }}=\int_{L^{t}}^{L_{m}} K e^{-\alpha z} d z=-\frac{K}{\sigma}\left[e^{\kappa L_{m}}-e^{\kappa L^{I}}\right],
$$

where $K$ is maximum root length density near soil surface $(z=0 \mathrm{~cm}), \alpha$ is a parameter determining the rate of decay of root length density with soil depth $z(\mathrm{~cm})$.

The normalized root length density (Equation 1) for the $i$ th soil layer can be calculated as

$$
L_{\text {nrd }, i}=\frac{L_{m} L_{d, i}}{\sum_{k=1}^{\mathrm{n}} \Delta z_{k} L_{d, k}+L_{\text {bottom }}}
$$


where the $2^{\text {nd }}$ term of the denominator $\left(L_{\text {bottom }}\right)$ is calculated according to Equation 3. Also, we used the relation $\int_{0}^{L_{m}} L_{d}(z) d z / L_{m}=\int_{0}^{1} L_{d}\left(z_{r}\right) d z_{r_{0}}$ Figure 1 shows the distribution of normalized root length density at different relative depths of soil.

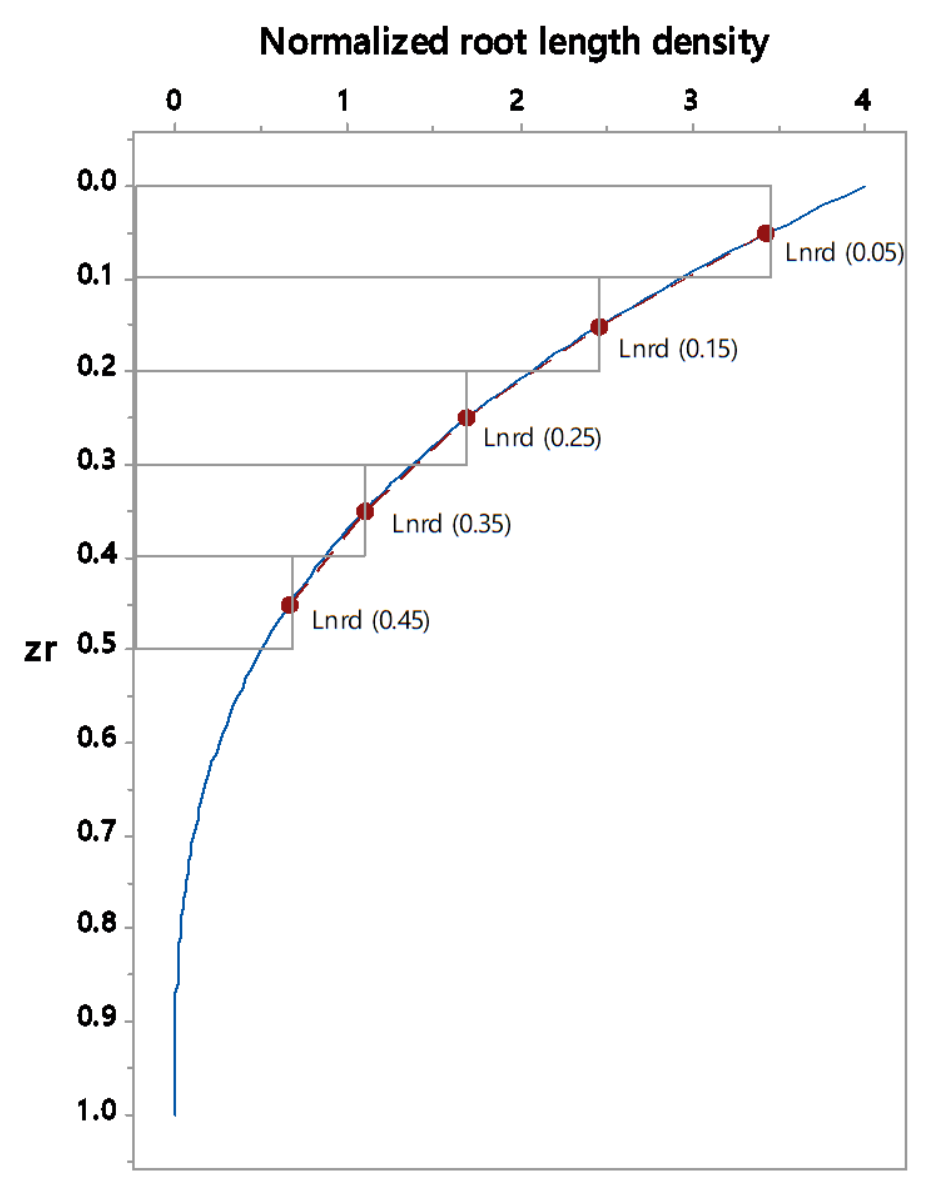

Figure 1. Normalized root length density $\left(L_{n r d}\right)$ as a function of relative soil depth $\left(z_{r}\right)$, based on measured root length densities in the upper root zone (rectangles). Numerals enclosed in parentheses indicate the relative depths of centers of the soil depth intervals with root measurement. Also shown is the predicted distribution of $L_{n r d}$ in the root zone, assuming $\beta=3.0$

The normalized root length densities calculated in Equation 4 will be fitted to a oneparameter nonlinear model of Ojha and Rai (1996), assuming maximum rooting depth $\left(L_{m}\right)$ is known:

$$
L_{\text {nrd }}=(\beta+1)\left(1-z_{r}\right)^{\beta}
$$

where $\beta$ is the unknown parameter to be estimated using nonlinear least-squares. Compared with the four-parameter polynomial equation of Wu et al. (1999), Equation 5 is simpler, containing only one parameter. It attains the maximum value near soil surface when $z_{r}=0$; it gives zero value at bottom of the root zone $\left(z_{r}=1\right)$. Also, the integral of Equation 5 over the root zone is unity: $\int_{0}^{1} L_{n r d}\left(z_{r}\right) d z_{r^{*}}$ It should be noted that Equation 5 was also obtained independently from a different derivation by Zuo et 
al. (2004, 2013) as a generalized root length density function. It was used by Dong et al. (2010) to characterize plant root distribution pattern in a mixed-grass prairie. The best parameter can be estimated using nonlinear curve-fitting method based on measured root length density data.

Once the values of $\beta$ are estimated based on experimental data, Equation 5 can be employed to calculate the relative soil depth $z_{r, 95}$ (or absolute soil depth $z 95$ ) above which $95 \%$ of roots are found. Knowing how much deep into the soil profile should we dig in order to find $95 \%$ of roots will surely help our future root survey by concentrating our efforts in the depth of soil that is most important for root growth and function. We can estimate $z_{r, 95}$ by evaluating the following definite integral:

$$
\int_{0}^{z_{Z_{i}, 95}}(\beta+1)\left(1-z_{r}\right)^{\beta} d z_{r}=0.95 \text {, }
$$

which yields

$$
z_{r, 95}=1-e^{\frac{\operatorname{In}(0.05)}{\beta+1}}
$$

Using Equations 6 and 7, the absolute depth above which 95\% of plant roots are found (i.e., z95) can be calculated as $z_{95}=L_{m} z_{r, 95}$ *

\section{Computer code with sample data testing}

The root distribution parameters can be calculated using the Minitab macro len.mac, or the R script len_R.txt, as can be found in the Appendix. As a test of the computer code, a sample input data file, soil.csv, is provided and the output results are shown in out.csv. The main output results include (a) distribution pattern of root length densities as measured from soil coring (not normalized within the whole root zone; see the documentation of len.mac in the Appendix); (b) the ratio of total roots contained in the upper and lower profile (the dividing line between the upper and lower profile is set at $40 \mathrm{~cm}$ in the illustration, but this can be changed easily); (c) the distribution pattern of normalized root length density as measured from soil coring; and (d) the estimated depth of soil containing $95 \%$ of total root lengths. Parameter values estimated from the nonlinear regression procedure are accompanied by estimated standard errors. As can be seen from the sample output file out.csv, standard errors of the model parameters for some soil depths are quite high, primarily due to the small sample size (5 for each soil core in the testing data set). The errors could be reduced for larger sample sizes with soil layers divided into thinner segments. It has been found that root vertical distribution is strongly influenced by soil bulk density (Zhang et al., 2012). Thus, under some situations, such as one in which there exists a hard plow pan, root distribution can become more complex. In that case, different nonlinear models may be used in place of current ones (modifications may be made to lines marked with *** in len.mac), as well as some other minor changes to properly collect the outputs from new nonlinear models.

The current setup of the programs gives reasonable estimates of the average root distribution patterns of winter wheat varieties from a relatively large data set obtained in field conditions, as seen in Figure 2, in which fitted values for normalized root length densities for 240 soil core samples are compared against the measured ones. The root samples were collected in two seasons in 2016 and 2017 during the flowering stage 
from the same set of ten winter wheat varieties planted in two locations in Texas: Amarillo and Uvalde. The soil in Amarillo is clay loam (Xue et al., 2003) while that in Uvalde is clay (Sharma et al., 2014). Amarillo (35 52' N, $101^{\circ} 58^{\prime} \mathrm{W}$; elevation $1114 \mathrm{~m}$ ) is located in Texas High Plains, with an annual rainfall of $516 \mathrm{~mm}$, average temperature of $14.1^{\circ} \mathrm{C}$ and average wind speed of $6.4 \mathrm{~m} / \mathrm{s}$. Uvalde $\left(29^{\circ} 13^{\prime} \mathrm{N}, 99^{\circ} 45^{\prime} \mathrm{W}\right.$; elevation $283 \mathrm{~m}$ ) is located in the southwest of Texas (Wintergarden), with an annual rainfall of $594 \mathrm{~mm}$, average temperature $27.6{ }^{\circ} \mathrm{C}$ and average wind speed $3.1 \mathrm{~m} / \mathrm{s}$. Root samples were taken using a hydraulic soil corer from $1 \mathrm{~m}$ profile at $20 \mathrm{~cm}$ intervals. The samples were promptly stored in a $-20{ }^{\circ} \mathrm{C}$ freezer and later washed to obtain the fine roots, which were scanned into digital images and measured for root length using the WinRhizo software version 2013b (Reagent Instruments Inc., Quebec, Canada). Root length density was expressed as the total length of roots contained in unit soil volume $\left(\mathrm{cm} / \mathrm{cm}^{3}\right)$. The winter wheat plots were planted with a seeding rate of $5.8 \mathrm{~g} / \mathrm{m}^{2}$ and row spacing of $19 \mathrm{~cm}$ (see Liu et al., 2018 for detail).

(1200 depth points from 240 soil cores)

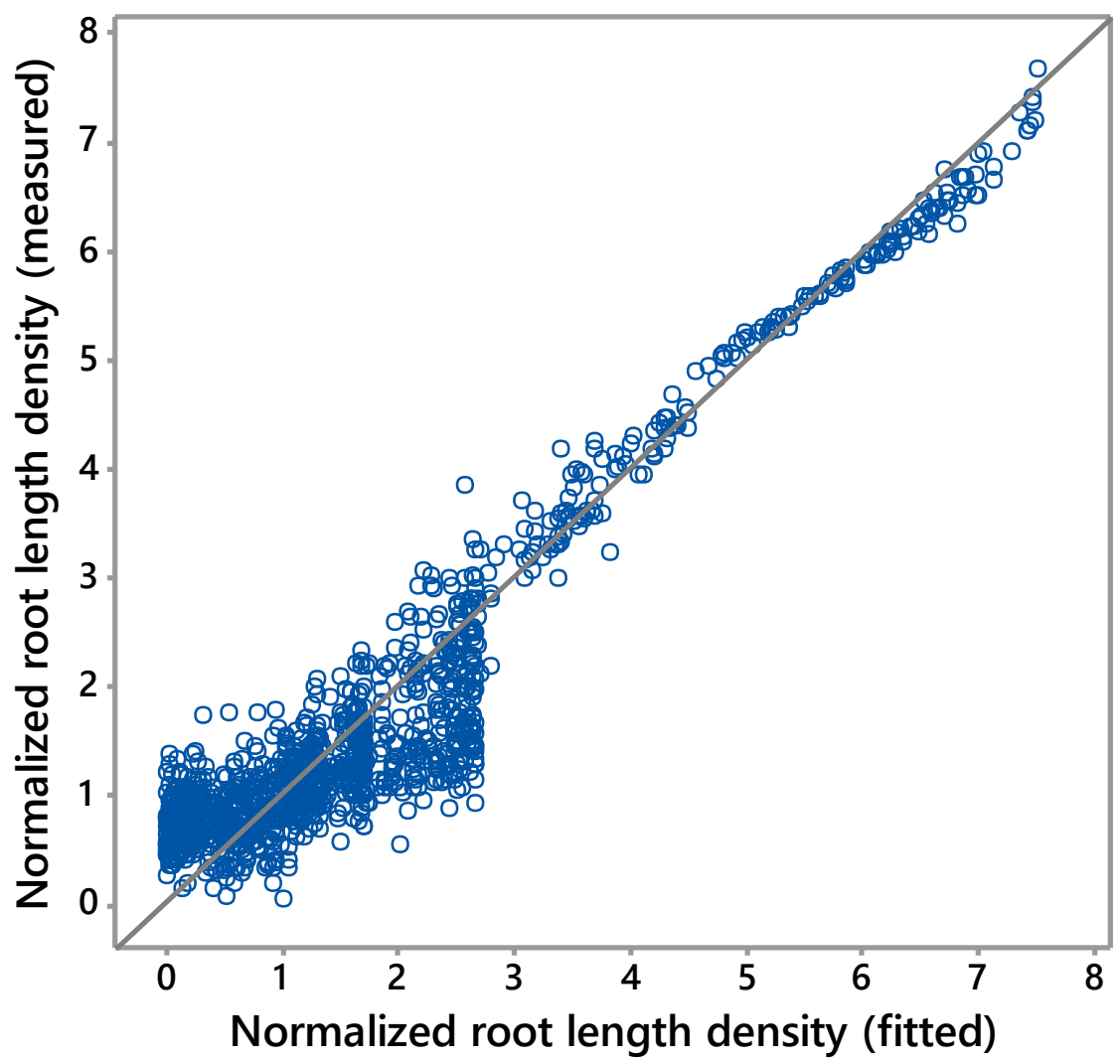

Figure 2. Measured normalized root length density $\left(L_{n r d}\right)$ as compared against the values fitted using the nonlinear regression based on 1200 depth points from 240 soil cores collected in winter wheat fields in Uvalde and Amarillo, Texas, USA. Also shown is the 1:1 line

The proposed root data processing method allowed us to estimate average soil depths (z95) containing 95\% of roots in two Texas locations. As seen in Figure 3, the average depth of $z 95$ in Amarillo was $117 \mathrm{~cm}$, while the value for Uvalde was only $63 \mathrm{~cm}$. A further discussion of the agronomic significance of these data is out of the scope of this 
method paper, but we do see clearly that the shallow rooting depths in Uvalde are associated with the heavy clay soil prone to compaction, while the soils in Amarillo are of clay loam type that promote deep root growth, especially for winter wheat (the dry, windy weather conditions in Amarillo may also be a factor influencing root distribution). Full data of root length density used to generate the results in Figures 2 and 3, as well as other related results, are collected in the Appendix. Finally, the computer code (both $\mathrm{R}$ script and Minitab macro), testing data and full data set are freely available at Zenodo (https://zenodo.org/record/1484655\#.W-o6BzFReUk).

Plant root distribution is frequently described by an empirical equation relating the cumulative proportion of roots to soil depth according to $Y=1-\beta^{D}$, where $Y$ is the cumulative proportion of roots (from 0 to 1 ), $D$ is soil depth (cm) and $\beta$ is a fitted extinction coefficient (Gale and Grigal, 1987; Jackson et al., 1996). While this equation can also be used to calculate the soil depth at which $95 \%$ of roots can be found, the parameter $\beta$ is defined differently from that in Equation 5, in which $\beta$ is associated with normalized root length density, a quantity that is directly useful for modeling root water uptake (Wu et al., 1999).

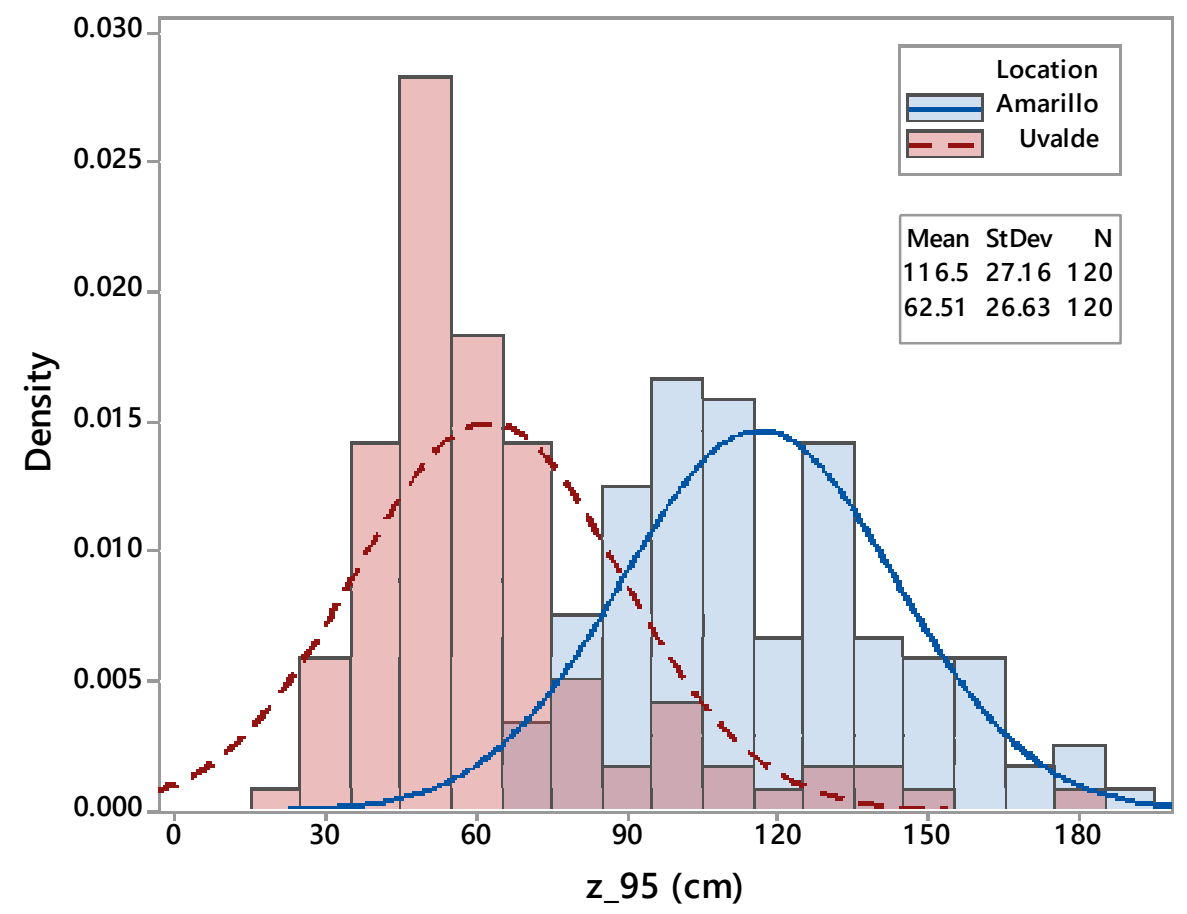

Figure 3. Histograms of soil depths that contain $95 \%$ of total root lengths ( $\left.z_{95}\right)$ for 240 plots of winter wheat growing in Amarillo and Uvalde of Texas, USA. The values of $z_{95}$ are calculated according to Equation 7 of the main text, which uses the estimated values of corresponding to each of the 240 soil core samples. "Density" in the vertical axis indicates probability density of the fitted normal distribution curves

\section{Implications and potential uses of the computer code}

Although root densities of plants generally tend to decrease exponentially from top to lower soil layers, the rate at which this decrease occurs may change under different situations. The computer code documented in this paper can facilitate a rapid extraction of parameters dictating root distribution patterns, with the potential of uncovering root 
traits associated with different genotypes, environment or management interaction scenarios. With the accumulation of field data of root distribution patterns in regional and global scales, rapid methods, such as the one proposed in this paper, are much needed in order to conduct meta-analysis using large data sets. In particular, the method proposed in this application note can handle variable sample sizes (one sample is defined as one set of roots obtained from one soil core divided into different segments), as well as flexible depth intervals (i.e., variable and/or uneven depth intervals for samples collected from the same ecosystem by different researchers under different circumstances). Finally, as the current method relies on the nonlinear regression procedure, the precision of parameter estimation is heavily dependent on the quality of field data (i.e., with soil cores sampled at fine, instead of coarse, segments).

Acknowledgements. We thank Shane Sieckenius and Dalton Thompson at the Uvalde Research Center and Kirk Jessup at the Amarillo Research Center for field assistance. We also thank Cathy Akritas at Minitab, Inc. for helpful suggestions for the development of the Minitab macro. The work was supported by the Texas A\&M AgriLife Research cropping system seed grant entitled "Root-shoot phenotyping and water balance characterization to improve water use efficiency and productivity of cropping systems in Texas", and by the USDA National Institute of Food and Agriculture NIFA Hatch project 1018758.

\section{REFERENCES}

[1] Acuña, T. L. B., Wade, L. J. (2013): Use of genotype $\times$ environment interactions to understand rooting depth and the ability of wheat to penetrate hard soils. - Annals of Botany 112: 359-368.

[2] Coupland, R. T., Johnson, R. E. (1965): Rooting characteristics of native grassland species in Saskatchewan. - Journal of Ecology 53: 475-507.

[3] Dong, X. (2016): How to put plant root uptake into a soil water flow model? F1000Research 5: 43. doi: 10.12688/f1000research.7686.1

[4] Dong, X., Patton, B. D., Nyren, A. C., Nyren, P. E., Prunty, L. D. (2010): Quantifying root water extraction by rangeland plants through soil water modeling. - Plant and Soil 335: 181-198.

[5] Gale, M. R., Grigal, D. F. (1987): Vertical root distribution of northern tree species in relation to successional status. - Canadian Journal of Forest Research 17: 829-834.

[6] Hodgkinson, L., Dodd, I. C., Binley, A., Ashton, R. W., White, R. P., Watts, C. W., Whalley, W. R. (2017): Root growth in field-grown winter wheat: Some effects of soil conditions, season and genotype. - European Journal of Agronomy 91: 74-83.

[7] Jackson, R. B., Canadell, J., Ehleringer, J. R., Mooney, H. A., Sala, O. E., Schulze, E. (1996): A global analysis of root distributions for terrestrial biomes. - Oecologia 108: 389-411.

[8] Liu, X., Dong, X., Xue, Q., Leskovar, D. I., Jifon, J., Butnor, J. R., Marek, T. (2018): Ground penetrating radar (GPR) detects fine roots of agricultural crops in the field. Plant and Soil 423: 517-531.

[9] Ojha, C. S. P., Rai, A. K. (1996): Nonlinear root water uptake model. - Journal of Irrigation and Drainage Engineering 122: 198-201.

[10] R Core Team (2018): R: A Language and Environment for Statistical Computing. - R Foundation for Statistical Computing, Vienna, Austria. https://www.R-project.org/.

[11] Sharma, S. P., Leskovar, D. I., Crosby, K. M., Volder, A., Ibrahim, A. M. H. (2014): Root growth, yield, and fruit quality responses of reticulatus and inodorus melons (Cucumis melo 1.) to deficit subsurface drip irrigation. - Agricultural Water Management 136: 75-85. 
[12] Wan, C., Xu, W., Sosebee, R. E., Machado, S., Archer, T. (2000): Hydraulic lift in drought-tolerant and-susceptible maize hybrids. - Plant and Soil 219: 117-126.

[13] Wasson, A. P., Rebetzke, G. J., Kirkegaard, J. A., Christopher, J., Richards, R. A., Watt, M. (2014): Soil coring at multiple field environments can directly quantify variation in deep root traits to select wheat genotypes for breeding. - Annals of Botany 65: 62316249.

[14] Weaver, J. E. (1926): Root Development of Field Crops. - McGraw-Hill Book Company, Inc., New York.

[15] Wu, J., Zhang, R., Gui, S. (1999): Modeling soil water movement with water uptake by roots. - Plant and Soil 215: 7-17.

[16] Xue, Q., Zhu, Z., Musick, J., Stewart, B., Dusek, D. (2003): Root growth and water uptake in winter wheat under deficit irrigation. - Plant and Soil 257: 151-161.

[17] Yadav, B. K., Mathur, S., Siebel, M. A. (2009): Soil moisture dynamics modeling considering the root compensation mechanism for water uptake by plants. - Journal of Hydrological Engineering 14: 913-922.

[18] Yu, G. R., Zhuang, J., Nakayama, K., Jin, Y. (2007): Root water uptake and profile soil water as affected by vertical root distribution. - Plant Ecology 189: 15-30.

[19] Zhang, X., Shao, L., Sun, H., Chen, S., Wang, Y. (2012): Incorporation of soil bulk density in simulating root distribution of winter wheat and maize in two contrasting soils. - Soil Science Society of America Journal 76: 638-647.

[20] Zuo, Q., Jie, F., Zhang, R., Meng, L. (2004): A generalized function of wheat's root length density distributions. - Vadose Zone Journal 3: 271-277.

[21] Zuo, Q., Zhang, R., Shi, J. (2013): Characterization of the Root Length Density Distribution of Wheat Using a Generalized Function. - In: Timlin, D., Ahuja, L. R. (eds.) Enhancing Understanding and Quantification of Soil-Root Growth Interactions. Chapter 5. Volume 4 of Advances in Agricultural Systems Modeling. ASA-CSSA-SSSA, Madison, WI, USA, pp. 93-117.

\section{ELECTRONIC APPENDICES}

1. List of Minitab macro len.mac.

2. List of R script len_R.txt.

3. Sample input data soil.csv.

4. Sample output results out.csv.

5. Full data set for generating results in Figures 2 and 3 (full.csv).

6. Output results from the full data set (full_out.csv). 